E.L.U.A. 2, 1984 , págs. $171-199$

\title{
NEGACIÓN Y ELIPSIS
}

\author{
IGNACIO BOSQUE \\ (Universidad Complutense. Madrid)
}

1. En ninguna de las cuatro o cinco monografías que existen sobre la negación en español se analiza el comportamiento de las estructuras negativas en los procesos sintácticos de elisión. Al no existir, además (al menos, cuando escribimos estas líneas), una monografia que estudie en profundidad las complejidades de la elipsis en nuestro idioma, la cuestión que nos ocupa queda prácticamente inédita en los estudios de gramática española, aunque es cierto que ha interesado a numerosos investigadores en otras lenguas. Por la extraordinaria amplitud de la cuestión, que sobrepasa con mucho las posibilidades de un articulo breve, como es éste, abordaremos únicamente algunas de las construcciones en las que intervienen ambas categorias. Estudiaremos la elipsis de sintagma verbal negado en las coordinadas y yuxtapuestas, asi como un tipo de construcción negativa interesante, que pudiera interpretarse falsamente como una elisión verbal.

Las numerosas investigaciones que se han realizado sobre la elipsis en algunas lenguas, en particular la inglesa, coinciden (independientemente de las diferencias metodológicas que puedan existir entre ellas) en algunos puntos significativos. En primer lugar, los constituyentes que se eliden están sujetos a constricciones formales diferentes que no permiten, frente a la visión un tanto intuitiva de la gramática tradicional, elidir cualquier información por el solo hecho de que se repita. En segundo lugar, existen clases de elipsis muy diferentes. Unas pertenecen a la gramática de la oración y otras a la del discurso; unas pueden 
darse únicamente en determinadas estructuras (por ejemplo, coordinadas), y otras se extienden a diálogos y aun a textos articulados mediante principios pragmáticos dificilmente formalizables. Algunos gramáticos de orientación generativista discuten en la actualidad acerca de si ciertos casos aparentes de elipsis deben reflejarse en la gramática mediante reglas interpretativas y no a través de procesos de borrado o supresión de unas informaciones previamente presentes de forma explícita. Algunos piensan que ciertos procesos oracionales de elisión deben realizarse en el plano que se ha dado en llamar la 'forma lógica' de la gramática en lugar de efectuarse a través de transformaciones de supresión. En este trabajo no entraremos a analizar directamente estas opciones, pero sí nos detendremos a considerar la naturaleza de los factores que regulan algunos tipos de elipsis, así como los criterios formales y semánticos que aconsejan no hablar de elipsis en otras situaciones aparentemente similares. La elipsis es uno de los muchos fenómenos sintácticos de la gramática española que se suelen 'reconocer' o 'etiquetar' sin demasiada dificultad, pero cuyos límites, constricciones, alcance y funcionamiento nos son todavia desconocidos.

2. Estudiaremos en este apartado la elisión de SV en las oraciones negativas. Como es sabido, dicha elisión es particularmente frecuente en las coordinadas (repetimos entre corchetes el sintagma suprimido):

(1) a. Juan confía en María, pero yo no $\varnothing$. [ $\varnothing=$ confío en María $]$

b. ¿Vienes o no $\varnothing$ ? $[\varnothing=$ vienes $]$

c. Tanto si piensas hablar con él como si no $\varnothing \cdot[\varnothing=$ piensas hablar con él]

d. No sé qué contarle y qué no $\varnothing$. $\lceil\varnothing=$ contarle $]$

e. Juan trabaja o no $\varnothing$ según le apetece. $[\varnothing=$ trabaja]

La elipsis de SV es igualmente posible en estructuras yuxtapuestas e incluso en diálogos. El mismo ejemplo (1 a) nos puede servir como muestra:

(2) Juan confia en María. Yo no. (Yuxtaposición)

(3) A: Juan confía en María.

B: Yo no.

(Diálogo)

Sin embargo, sería un error pensar que la elipsis de SV que reconocemos en (1), (2) o (3) es la misma que podemos identificar en las respuestas constituidas únicamente por el adverbio no. Frente al análisis, absolutamente generalizado, que interpreta las respuestas negati- 
vas (¿Hace frío? / No; ¿Llovió mucho? / No) como casos de elipsis, algunos autores han apuntado recientemente que tales respuestas no deben analizarse como resultados de procesos de elisión. C. Plantin (1982) observa, por ejemplo, que las relaciones deícticas se alteran en tales preguntas, to que hace muy difícil postular un elemento previo que haya sido suprimido. Junto a los ejemplos impersonales citados, no hay elipsis, argumenta Plantin, al responder No a ¿Quieres un poco? (no elidiriamos quieres sino quiero) o a ¿Vienes? (no elidiríamos vienes ni verigo sino voy). Si la elipsis es, esencialmente, la supresión de cierta información que ha aparecido antes en la oración o en el discurso, es sumamente problemático identificar esa información en diálogos como los de (4):

(4) A: ¿Vendrás tú aquí esta tarde?

B: No.

ya que, salvo esta tarde, los elementos que considerariamos elididos (yo, iré, alli) no han aparecido en el diálogo citado. La argumentación de Plantin nos parece correcta '. Para comprobar la diferencia que existe entre estructuras como las de (1) y las (4) comparemos las dos oraciones de (5):

(5) a. ¿Vienes? / No.

b. Tú quieres venir, pero yo no $\varnothing$.

Nótese que, mientras que interpretariamos el no de $(5 \mathrm{a})$ en el sentido de no voy, y no en el de no vengo, hariamos exactamente lo contrario en ( $5 \mathrm{~b}$ ). Dicha oración no equivale a Tú quieres venir pero yo no quiero ir, sino a Tú quieres venir pero yo no quiero venir. Las oraciones de (6) muestran una alternancia similar:

(6) a. ¿Sabes si va a venir María? / Sí.

b. Tú no sabes si va a venir Maria, pero yo sí.

Mientras que el adverbio Si de (6 a) admite una doble interpretación

1 C. Plantin proporciona otros muchos argumentos en su trabajo, pero no entraremos a analizarlos. Si contestamos, por ejemplo. Si, Pedro y Maria a la pregunta ¿Sabes quién vendrá?, la interpretación eliptica nos obligaria a suponer una oración como Sé quién vendrá: Pedro y Maria, pero la respuesta anterior parece equivaler más bien a Sé que Pedro y María vendrán, que no puede obtenerse por ningún proceso de elipsis. 
contextual ("Sé si va a venir María» o "va a venir María»), no hay tal ambigüedad en (6 b). La lectura más plausible en (6 a), es decir, "va a venir María", es precisamente la que no estamos autorizados a obtener en ( 6 b), ya que en esta oración elidimos un SV y no buscamos la interpretación contextual de una respuesta de acuerdo con la información que nos proporciona el discurso ${ }^{2}$.

Aunque las estructuras que hemos analizado poseen, claramente, una sintaxis diferente, en la elipsis de SV no siempre se reproduce fielmente la información que permanece en la secuencia. Así, como se muestra en (7 a), puede darse una alteración de género en el proceso de elisión, y como se observa en $(7 \mathrm{~b})$ y $(7 \mathrm{c})$, también el número y la persona resultan alterados.

(7) a. María es catalana, pero Pablo no $\varnothing$. [ $\varnothing=$ es catalán]

b. Luis y Ana están casados, pero Pedro no $\varnothing .[\varnothing=$ está casado $]$

c. Yo tengo frío, pero tú no $\varnothing[\varnothing=$ tienes frio $]$

Finalmente, la 'identidad de sentido' puede sustituir a la 'identidad de referencia' en la elipsis de SV. Utilizaremos los índices «i indicar correferencia:

(8) Juan confía en $s u_{i}$ médico, pero Pedro, no $\varnothing$.

$\mid \varnothing=$ confia en $s u_{\mathrm{j}}$ médico $]$, o bien $\left[\varnothing=\right.$ confia en $s u_{\mathrm{i}}$ médico $\mid$

A pesar de esta aparente libertad, la elipsis de SV en cláusulas negativas está fuertemente restringida, tanto desde el punto de vista semántico como sintáctico:

2 No existen muchos estudios sobre la sintaxis y semántica del adverbio si. Véanse Dumitrescu (1973 y 1979) y Carbonero Cano (1980). Nos parece que deben separarse claramente los dos valores que ejemplificamos en $(6$ a) y ( 6 b). Este último uso de si mantiene una interesante relación discursiva con el texto inmediatamente precedente. Si consideramos las tres respuestas que se indican en (i)

(i) a. Yo.

b. Yo no.

c. Yo si.

veremos que (i a) y ( $\mathrm{i}$ b) son contestaciones apropiadas a la pregunta ¿Quién quiere ir?, pero (i c) sólo lo sería si el hablante tuviera razones para deducir del contexto extralingüístico que alguien más no desea ir. Por el contrario, (i a) y (i c) son respuestas apropiadas a la pregunta ¿Quién no quiere ir?, mientras que $(i b)$ difícilmente lo seria. 
La elisión no puede darse, en primer lugar, en las coordinadas de carácter enfático en las que sólo la negación diferencia un miembro del otro.

(9) a. Quiere hacerlo y no quiere hacerlo. No acaba de decidirse.

b. *Quiere hacerlo y no $\varnothing$. No acaba de decidirse.

Más significativas son las constricciones de naturaleza sintáctica. Como regla general, la supresión del SV que introduce la negación debe ser total. No es posible mantener uno de los miembros de dicho constituyente y elidir otro. En (10b) hemos realizado una elisión incorrecta. La única posibilidad en tales casos es realizar una sustitución, como se indica en (10c):

(10) a. Juan lee despacio, pero María no $\varnothing \cdot[\varnothing=$ lee despacio $]$

b. *Juan lee despacio, pero Maria no lee $\varnothing$. $[\varnothing=$ despacio $]$

c. Juan lee despacio, pero María no lee asi.

Idéntica conclusión se obtiene de las elisiones imposibles que se señalan en (11) y (12):

(11) a. "Maria parece enferma, pero Luisa no parece $\varnothing \cdot 1 \varnothing=$ enferma]

b. "María parece enferma, pero Luisa no $\varnothing$ triste. $\lceil\varnothing=$ parece $]$

(12) a. *Luis habló con Pedro, pero Antonio no habló $\varnothing \cdot 1 \varnothing=$ con Pedro]

b. *Juan ha estado buscando trabajo, pero Pedro no ha estado $\varnothing \cdot[\varnothing=$ buscando trabajo $]$

Los sintagmas de infinitivo subordinados a ciertos verbos (poder, deber, ser capaz, querer, etc.) pueden ser elididos en construcciones como las citadas. Compárense las elisiones correctas de (13) con las incorrectas de (14):

(13) a. Quisimos ir, pero no supimos $\varnothing \cdot[\varnothing=i r]$

b. Juan consiguió terminar su trabajo, pero Pedro no fue capaz $\varnothing \cdot 1 \varnothing=$ de terminar su trabajol

c. Juan no irá. Pedro, en cambio, debería $\varnothing \cdot[\varnothing=i r]$

(14) a. "María sintió hacerlo, pero Luisa no sintió $\varnothing \cdot[\varnothing=$ hacerlo]

b. *Juan dijo saber la causa, pero Antonio no dijo $\varnothing .1 \varnothing=s a-$ berla]

c. *Juan no irá. Pedro, en cambio, ha pensado $\varnothing \cdot[\varnothing=$ en ir $]$ 
Los predicados que permiten la elisión de su infinitivo en (13) son los mismos que aceptan la elipsis de SV no infinitivo en ciertas subordinadas adverbiales (las introducidas por cuando, si, como, donde, porque, etc.):

(15) a. El chico estudia porque su madre quiere $\varnothing \cdot[\varnothing=$ que estudie]

b. Le insisti en que viniera, pero no quería $\varnothing \cdot[\varnothing=$ venir $]$

(16) a. Hizo el trabajo como pudo $\varnothing \cdot[\varnothing=$ hacerlo $]$

b. Quiso hacer el trabajo, pero no pudo $\varnothing \cdot[\varnothing=$ hacerlo $]$

Los verbos que rechazan la elipsis de infinitivo en el complemento de un SV negado son también los que rechazan la elipsis de complemento verbal en las subordinadas citadas:

a. $\quad$ E El chico estudia porque su madre desea $\varnothing \cdot[\varnothing=$ que estudie $]$

b. "No hizo el trabajo porque no necesitaba $\varnothing . \mid \varnothing=$ hacerlo]

No entraremos a analizar con detalle las caracteristicas semánticas de los predicados que pueden aparecer en construcciones como las de (13), (15) o (16). En principio, parece claro el carácter modal de la mayoria de ellos ${ }^{3}$, pero debe observarse que verbos como gustar 0 saber no son, estrictamente hablando, verbos modales, si es que es posible definir con objetividad esta categoria en español (véase A. Narbona, 1981). Los verbos citados favorecen la elisión, al igual que predicados como parecer dispuesto, ser incapaz, mostrarse favorable o tener intención. En nuestra opinión, deberia investigarse detenidamente la relación que existe entre las elisiones citadas y los predicados que parecen expresar predisposición, actitud o propósito, aunque no sean verbos auxiliares. No debe ocultarse, sin embargo, que este tipo de elisiones entrarian, al menos en parte, en el grupo de las que están regidas por principios pragmáticos, y no por factores estrictamente sintácticos o incluso semánticos. Asi, Hankamer y Sag (1976) proponen oraciones como Pedro aceptó o Luis no estaba de acuerdo como ejemplos de relaciones anafóricas controladas pragmáticamente. En ellas, "there is no evidence that a real complement was ever present to un-

"No tenemos, como es sabido, "/o pudo en (16) ni "lo queria en (15 b) ni "lo deberia en (13 c), y si, en cambio, lo desea en (17 a) o lo necesita en (17 b). Esta diferencia no es, sin embargo, demasiado útil para estudiar complementos preposicionales como los de (13 b) o (14 c). Cf. la nota 10 . 
dergo any syntactic rule in the matrix clause». De hecho, los mismos autores apuntan que oraciones como Maria se ha quejado a los vecinos pueden ser usadas sin que exista un contexto verbal previo. (Basta, por ejemplo, con que nuestro interlocutor haya oído el mismo ruido que nosotros o que perciba lo que le mostramos ante sus ojos.) La elipsis que pueda darse en tales contextos no es una operación sintáctica en el sentido de que no son factores sintácticos los que regulan la ausencia de la información que se considera suprimida.

Existe otro factor, éste claramente sintáctico, que condiciona poderosamente las elisiones de SV. J. Martínez Alvarez (1978) ha estudiado algunas elipsis interesantes en las construcciones de «verbo auxiliar + infinitivo». Estos son sus ejemplos:

(18) a. ¿Se puede beber esta botella?

b. ¿Se debe pagar a los colaboradores?

c. ¿Se suele beber vino?

(19) a. ¿Se pueden beber estas botellas?

b. ¿Se deben pagar estos trabajos?

c. ¿Se suelen cantar canciones?

La autora citada observa, correctamente, que es posible contestar, respectivamente, No se puede, No se debe o No se suele a las oraciones de (18), pero no lo es contestar No se pueden, No se deben o No se suelen a las oraciones de (19). Concluye Martínez Alvarez que estas botellas es sujeto en (19a), pero no en (18a). El sujeto de esta última oración es beber esta botella. A menos que contestemos únicamente si o no a las preguntas de (18) o (19), reproduciremos en ambos casos todo el predicado verbal. En (18a), el predicado es se puede, ya que el sujeto es beber esta botella, pero en (19a) el predicado es se pueden beber, ya que el sujeto es estas botellas.

La elisión de SV que estamos estudiando no debe confundirse con el tipo de elipsis en la coordinación o la yuxtaposición que en la bibliografía generativista se conoce con el nombre de 'gapping'. Dicho proceso consiste en elidir algunos de los constituyentes del SV contiguos al verbo (nunca todos) como se indica en (20) ${ }^{4}$ :

4 Existe una amplisima bibliografía sobre este tipo de elipsis, sobre todo en la gramática inglesa. Véanse, entre otros, Ross (1967), Jackendoff (1971), Hankamer (1971 y 1973), Stillings (1975), Kuno (1976a) y, recientemente, Neijt (1979). 
(20) Juan piensa en irse de vacaciones a Italia este verano, y Pedro $\left\{\begin{array}{l}\varnothing \text { en irse de vacaciones a Francia este otoño. }[\varnothing=\text { piensa }] \\ \varnothing \text { a Francia este otoño. [ } \varnothing=\text { piensa en irse de vacaciones] } \\ \varnothing \text { este otoño. }[\varnothing=\text { piensa en irse de vacaciones a Italia] }\end{array}\right\}$

Existen notorias diferencias sintácticas entre este tipo de elisión y la elipsis de SV, pero no podremos detenernos aqui a analizarlas con detalle (véase Sag (1977) para un estudio comparativo). Indicaremos, no obstante, algunas particularidades por lo que respecta a la negación:

En primer lugar, la elipsis parcial de SV en coordinadas a las que nos referimos es dificil de realizar en SSVV negados, a menos que se altere el orden de los constituyentes (véanse más adelante las oraciones de (41)).

(21) a. ?? Juan confia en Maria, pero María no $\varnothing$ en Juan. $\varnothing \varnothing=$ confia]

b. Juan confía en Maria, pero Maria en Juan no $\varnothing \cdot 1 \varnothing=$ confia]

Aunque pudiera pensarse que esta diferencia se debe, simplemente, a la presencia de una conjunción adversativa, ya que no tenemos habitualmente "Juan habló con Pedro, pero éste $\varnothing$ con Antonio (cf. ...y éste...), lo cierto es que las coordinadas copulativas todavía son más reticentes a aceptar elisiones como las de (21 a):

(22) a. *Antonio realizó su tarea y Pedro no $\varnothing$ la suya. $[\varnothing=$ realizó $]$

b. *Juan llegó tarde al colegio y, aunque no lo creas, María no $\varnothing$ al trabajo. $[\varnothing=$ llegó tarde]

Varios autores (Ross (1967), Jackendoff (1971), Stillings (1975, nota 8) y Sag (1977: 143 y sig.)) han observado que, aunque podemos tener dos verbos negados en los dos miembros de una coordinación, es imposible elidir el segundo de ellos. No existe más solución que repetir el verbo, como en ( 23 a), o utilizar una conjunción negativa, como en $(23 \mathrm{c})$ :

(23) a. Juan no entendió a Pedro, y Luis no entendió a Maria.

b. "Juan no entendió a Pedro, y Luis no $\varnothing$ a María. $1 \varnothing=$ entendió ]

C. Ni Juan entendió a Pedro ni Luis $\varnothing$ a María. $\mid \varnothing=$ entendió $\mid$ 
(24) a. O el tren no llegaba a tiempo o el autobús no llegaba a su hora.

b. *O el tren no llegaba a tiempo o el autobús no $\varnothing$ a su hora. $\varnothing \varnothing=1$ legaba]

c. Ni el tren llegaba a tiempo ni el autobús $\varnothing$ a su hora. $\lceil\varnothing=$ llegaba]

La imposibilidad de realizar, como muestran (23 b) y (24 b), una elipsis parcial de SV en coordinadas copulativas negadas ${ }^{5}$ no se extiende en español a un tipo particular de estructuras que son posibles gracias a la doble negación caracteristica de nuestra lengua:

(25) a. Pedro tiene dos hermanas, y Luis $\varnothing$ ningún hermano.

b. Antonio fue a Italia, y Pedro $\varnothing$ a ningún sitio.

c. Juan confía en mucha gente. María $\varnothing$ en nadie.

Nótese que en todos los casos " $\varnothing$ " debe incorporar un verbo negado, ya que en español no son aceptables los pronombres y adverbios negativos en posición postverbal si el verbo no está negado ( ${ }^{*} P e d r o$ fue a ningún sitio, "Maria confia en nadie, etc. (cf. Bosque (1980, cap. 2))). Existe una posible explicación para estos hechos, que se basa en el carácter recuperable (por redundante) de la negación preverbal. I. Sag argumentaria, probablemente, que este tipo de fenómenos apoya la hipótesis de que tal proceso de elipsis se realiza en el nivel de la forma lógica, donde no existen negaciones redundantes. Muy similar es el problema de interpretar " $\varnothing$ " en oraciones como Pepe quiere algo, pero yo no $\varnothing$. ya que la presencia de una negación preverbal nos exigiría entender " $\varnothing$ " como "...quiero nada" y no como "...quiero algo".

La elisión en SSVV negados que estamos estudiando es un proceso inviable en estructuras subordinadas (salvo en las completivas, y sin embargo es frecuente en diversos tipos de coordinadas. Nótese que la

S. Kuno (1982: 148) explicaría tal vez estas alternancias como consecuencia de las constricciones que poseen muchas lenguas para construir secuencias con 'focos' negativos o cuasi-negativos (cf. más adelante nuestros ejemplos de (24) y siguientes). Kuno cree, por tanto, que no es casual que la oración ( $i b$ ) sea incorrecta, o al menos extraordinariamente forzada (los ejemplos originales están en inglés):

(i) a. Juan sabe mucho de matemáticas, y Luis $\varnothing$ de lingüística.

b. "Juan sabe poco de matemáticas, y Luis $\varnothing$ de lingü ística.

Véase el §3.3.3.1 de Bosque (1980) acerca del valor negativo de poco. 
elisión no puede darse en oraciones como las de (26), que pueden compararse con las elisiones correctas de (1):

(26) a. *María iba al cine cuando Luisa no $\varnothing$. [ $\varnothing=i b a$ al cine $]$

b. "Yo hablé del asunto porque Juan no $\varnothing \cdot[\varnothing=$ habló del asunto]

c. ${ }^{*}$ Prefiero ir donde tú no $\varnothing .[\varnothing=$ vayas $]$

d. "Maria dice que irá si Pedro no $\varnothing \cdot[\varnothing=v a]$

Kato (1981: 212) observa con ejemplos portugueses que las subordinadas temporales y condicionales no admiten la elisión del verbo en la principal. La situación es idéntica en español:

(27) a. *Si Juan toma café por la mañana, $\varnothing$ té por la tarde. $\varnothing \varnothing=$ toma ]

b. ${ }^{\star}$ Cuando veo a Luis, $\varnothing$ también a su hermano. $[\varnothing=$ veo $]$

Es bien sabido que existen conjunciones que poseen una función coordinante y otra subordinante. Un ejemplo paradigmático es la conjunción aunque (véase Lázaro Mora, 1982). La elisión de SV es posible si aunque posee la primera función, pero no si posee la segunda:

(28) a. Yo pienso hablar con él; aunque Juan, no $\varnothing \cdot[\varnothing=$ piensa hablar con él]

b. "Yo pienso hablar con él aunque Juan no $\varnothing \cdot \varnothing \varnothing=$ piense hablar con él]

Debe tenerse en cuenta que en la interpretación de " $\varnothing$ " en (28 a) encontramos un verbo en indicativo, como corresponde al uso adversativo de aunque, pero en la interpretación de ( 28 b) encontramos un verbo en subjuntivo, ya que aunque es en dicha oración una conjunción concesiva. Una alternancia muy similar nos la proporciona el par mientras - mientras que. Cuando mientras posee un valor temporal, la elisión no es posible, como se observa en ( 29 a), pero sí lo es cuando posee un valor adversativo ('contrastivo' para algunos gramáticos), como en (29 b), es decir, cuando no es una conjunción subordinante:

(29) a. *Juan estudiaba mientras tú no $\varnothing \cdot[\varnothing=$ estudiabas $]$

b. Juan estudiaba, mientras que tú, no $\varnothing \cdot[\varnothing=$ estudiabas $]$

La elipsis de SV es, igualmente, inviable en las estructuras sintácticas que los gramáticos generativistas llaman «islas» porque no es po- 
sible extraer de ellas ningún sintagma mediante una regla de movimiento. Los SSNN complejos (sustantivos con complementos preposicionales o subordinadas relativas) pertenecen a este grupo. La elisión de SV no es posible en tales contextos, como se observa en las siguientes oraciones (en cursiva los SSNN complejos):

(30) a. Maria pensaba ir, pero mencionó que Juan no $\varnothing$.

b. "María pensaba ir, pero mencionó el hecho de que Juan no $\varnothing$.

a. Juan ha leido algunas novelas policiacas, pero Pedro no $\varnothing$.

b. *Juan ha leido algunas novelas policiacas que Pedro no $\varnothing$.

La elipsis de SSVV negados incluidos en SSPP es posible si éstos van regidos por adjetivos o verbos que cumplan las condiciones semánticas, o tal vez pragmáticas, a las que aludíamos más arriba (cf. (13) y (14), si es que esos son realmente casos de elisión sintáctica), pero parece muy dificil llevarla a cabo en el resto de las situaciones:

(32) a. *Maria trabajaba para que su hijo no $\varnothing \cdot[\varnothing=$ trabajara $]$

b. "Yo no miré hasta que él no $\varnothing$. $\varnothing \varnothing=$ miró $]$

La oposición entre preposiciones y conjunciones nos parece particularmente significativa para estudiar los contextos en los que se permite la elisión de SV, incluso en estructuras no negativas. Nótese que antes funciona como conjunción (antes que) cuyo valor comparativo, más que el estrictamente temporal, le permite elidir el SV de la oración que introduce. Antes de, por el contrario, es una locución prepositiva, y no conjuntiva, luego no es de esperar que la elipsis se permita en el término de dicha preposición, como en los ejemplos de (32). La diferencia se observa claramente en (33):

a. Luis habló con ella antes que Pedro $\varnothing$. [ $\varnothing=$ hablara con ella $]$

b. "Luis habló con ella antes de que Pedro $\varnothing$. $[\varnothing=$ hablara con ella]

La clase semántica a la que pertenezca el verbo principal en las estructuras que venimos analizando desempeña un papel esencial en la elipsis de SV que aquí nos interesa. J. O. Urmson observó en 1952 (aunque la idea cuenta con seguridad con precedentes) que existe un paradigma de verbos que admiten una doble interpretación contextual, según predomine o no el contenido proposicional de su subordinada sobre la aserción misma que se realiza al utilizarlos. Si alguien dice, por ejemplo, Creo que ha ganado Pedro, es posible que obtenga como 
réplica una refutación del contenido de la proposición subordinada (No, no ha ganado) o una refutación de la afirmación misma (No estoy convencido de que creas eso). Urmson llamaba 'parentéticos' a estos verbos porque pueden aparecer en la posición final de la oración en incisos que se conocen con tal nombre (Ha ganado Pedro, creo yo; Luis es muy astuto, recuerda). A este paradigma pertenecen verbos como creer, recordar, suponer, considerar, parecer, figurarse, a veces decir, etc. Muchos gramáticos se refieren a ellos como «verbos de afirmación débil», siguiendo la denominación de J. B. Hooper (1974) que, en su penetrante estudio de estos predicados, los oponía a los «verbos de afirmación categórica» (Strong assertive verbs) como insistir o mantener, que no poseen exactamente sus mismas propiedades, entre otras clases semánticas de predicados. Existe una notable coincidencia entre los 'verbos de afirmación débil' de Hooper o los 'parentéticos' de Urmson y los que P. Lysvag (1975) llama 'verbos de aproximación' (verbs of hedging), aunque este último autor no menciona dichas semejanzas. Consideremos ahora las dos oraciones de (34):

(34) a. Juan cree que Pedro está enfermo, pero María no $\varnothing$.

b. Juan siente que Pedro esté enfermo, pero María no $\varnothing$.

La oración ( 34 a) admite una doble interpretación según sustituyamos " $\varnothing$ " por «está enferma" o por "cree que Pedro está enfermo", de acuerdo con la doble lectura que favorecen los verbos de afirmación débil. " $\varnothing$ » en (34 b) sólo admite, por el contrario, la interpretación asertiva ${ }^{6}$ ( $\varnothing=$ siente que Pedro esté enfermo), ya que los verbos factivos, como sentir, rechazan la interpretación parentética a la que nos referimos ${ }^{7}$.

Pueden encontrarse otros reflejos sintácticos de la divergencia señalada. Consideremos brevemente las dos oraciones de (35):

a. Creo que Juan no asistió a la reunión de ayer.

b. Siento que Juan no asistiera a la reunión de ayer.

y fijémonos en las posibles réplicas Yo también y Yo tampoco. La primera de estas dos réplicas sería apropiada tanto para ( 35 a) como para $\left(35\right.$ b). Sin embargo, la oración ( 35 a) admite como posible réplica $Y_{O}$ tampoco ( = «Yo tampoco asistí»), pero (35 b) requiere la presencia del verbo: Yo tampoco asisti, y no Yo tampoco.

6 Nos referimos a esta cuestión, desde otro punto de vista, en el §2.4.2.2 de Bosque (1980).

La yuxtaposición se diferencia claramente de la coordinación en este punto. Asi, no es posible la interpretación parentética en Juan cree que Pedro está entermo. Maria no $\varnothing$. 
Creemos que existe una cierta relación entre el paradigma de verbos de "afirmación débil» y la propiedad que permite, en la lengua coloquial, elidir un SV negado en el segundo miembro de una coordinada adversativa:

(36) a. Pedro creía que llegaria a tiempo, pero no $\varnothing$.

b. Esperaba que llamaran, pero no $\varnothing$.

a. *Juan se entristeció por haber llegado tarde, pero no $\varnothing$.

b. "Me alegré de que María fuera a venir, pero no $\varnothing$.

A ello debe añadirse que es lógico que los verbos factivos (no asertivos) no funcionen en estructuras como las de (36) porque no se puede negar la información que se presupone cierta. Tales verbos rechazan también las coordinadas en las que la conjunción adversativa introduce un sintagma adverbial, adjetival o preposicional (semejante, en parte, a los que estudiaremos en el $\S 3$ ):

(38) a. Dijo que vendría, pero sólo.

b. Me parece que llegó, pero tarde.

c. Creo que aprobó, pero en septiembre.

(39) a. *Siento que viniera, pero sólo.

b. *Es extraño que llegara, pero tarde.

c. "Me alegro de que aprobase, pero en septiembre.

Para que las oraciones de (39) fueran correctas, los adverbios habrían de modificar al verbo principal, $y$, como por razones semánticas evidentes, ello no es posible, las oraciones quedan excluidas al no ser posible la referencia a la subordinada. (Nótese que el subordinante que puede aparecer delante de María en (34 a), pero difícilmente en (34 b).

Una última diferencia entre los predicados de afirmación débil (y quizá también en este caso los de afirmación categórica) y los factivos, asertivos o no, la encontramos claramente marcada en las estructuras en las que se produce una elipsis catafórica de SV, que sólo los primeros permiten:

(40) a. Antonio dice que no $\varnothing$, pero yo creo que volverá. $[\varnothing=\mathrm{vo}$ verá]

b. *Juan sintió mucho que no $\varnothing$, pero yo creo que tal vez hubiera podido venir. $[\varnothing=$ hubiera podido venir $]$

a'. María está convencida de que no $\varnothing$, pero yo creo que ha aprobado. $[\varnothing=$ ha aprobado $]$ 
b'. "Maria está triste porque no $\varnothing$, pero yo creo que debería haber aprobado. $[\varnothing=$ ha aprobado $]$

3. En el apartado anterior hemos estudiado la elipsis de SV en oraciones negativas coordinadas o yuxtapuestas. A pesar de su aparente similitud, dicho proceso no debe confundirse con el tipo de fenómenos que abordaremos en este apartado. Consideremos atentamente las dos oraciones siguientes:

(41) a. Juan confia en Luis, pero no en María.

b. Juan confía en Luis, pero en María no.

Un análisis superficial de estas oraciones podria hacernos pensar en una simple permutación de los constituyentes que aparecen en el segundo miembro de la coordinación. Sin embargo, si estudiamos ambas oraciones con mayor atención, veremos que su significado es muy distinto. La razón fundamental es que $(41 \mathrm{~b})$ es un ejemplo de elipsis verbal (aunque el orden de los constituyentes no sea el mismo que hemos visto en el §2), mientras que en ( 41 a) no se ha dado tal elisión ni ninguna otra. Trataremos de justificar, en primer lugar, esta última afirmación:

a) La ausencia de elipsis en oraciones como (41 a) se percibe más claramente si añadimos otros constituyentes al predicado verbal. Los ejemplos de (42) deberian ser sinónimos si estuviéramos ante un caso de elipsis, pero es evidente que no lo son:

(42) a. Pedro invitó a Maria a cenar y no invitó a su hermana.

b. Pedro invitó a María a cenar, y no a su hermana.

Nótese que de suponer un proceso de elipsis en estructuras como las que comentamos, habríamos de derivar oraciones como Llegaremos el jueves, y no el viernes de secuencias absurdamente redundantes como Llegaremos el jueves y no llegaremos el viernes.

b) La elipsis de SV en oraciones coordinadas puede realizarse en estructuras catafóricas con numerosas restricciones, algunas de las cuales vimos ya en el $\S 2$. Las oraciones que ahora nos ocupan no están sujetas a esas mismas restricciones, como se muestra en (43):
a. ??Juan no $\varnothing$, pero Pedro si es el verdadero culpable.
b. Juan, y no Pedro, es el verdadero culpable. 
No se puede suponer ningún elemento elidido entre no y Pedro en (43b).

c) El carácter no oracional del constituyente no en María en (41 a) inhabilita a este tipo de construcción para aparecer en periodizaciones de estructuras proposicionales. Las oraciones de (44) muestran claramente esta diferencia entre los dos tipos de estructura que nos interesan, a pesar de la dificultad que existe para reflejar las pausas en la lengua escrita:

(44) a. Vino Juan, no Pedro.

b. Vino Juan. Pedro no.

(45) a. "Vino Juan. No Pedro.

b. "Vino Juan, Pedro no.

De igual forma, la secuencia Pedro no podría servir de réplica o respuesta apropiada a una afirmación o a una pregunta precedente, pero no Pedro difícilmente lo sería en ninguno de los dos casos.

d) Otro argumento que prueba la ausencia de elipsis en oraciones como ( 41 a) lo podemos obtener de las secuencias en las que dicha estructura se combina con un verdadero caso de elisión:

(46) Juan viajó a Madrid, y María $\varnothing$ a Barcelona, y no Juan a BarceIona y Maria a Madrid.

En (46) tenemos una sola elipsis y no tres procesos de elisión. Nótese que, frente al caso de (42), aquí ni siquiera sería posible reconstruir los elementos supuestamente elididos sin alterar gravemente la sintaxis de la oración.

e) Finalmente, la teoría de los actos verbales puede darnos una última confirmación del carácter no derivado de las estructuras que comentamos, y por tanto, de la ausencia de constituyente verbal en las combinaciones $N o+S N$ y No $+S P$ que venimos analizando. Es fácil comprobar que al usar oraciones como (47 a) estamos realizando dos promesas, pero al emplear ( $47 \mathrm{~b}$ ) realizamos una única promesa:

(47) a. Te prometo que estudiaré con Pablo y que no estudiaré con María.

b. Te prometo que estudiaré con Pablo, y no con María.

Si (47 b) fuera el resultado de realizar un proceso de elisión en (47 a), no tendriamos en absoluto esa diferencia. 
Desde el punto de vista semántico existe una importante diferencia entre (41 a) y (41 b) o entre (42 a) y (42 b). Llamaremos "correctivas"a las negaciones que aparecen en ( $41 \mathrm{a}),(42 \mathrm{~b})$ y (46) usando un término que introdujo A. Bello (1847) para estructuras no demasiado alejadas de las nuestras ". Las negaciones correctivas presuponen que la proposición que puede 'reconstruirse' (no 'elidirse') en los sintagmas en los que aparecen puede ser considerada cierta, o al menos esperable, de acuerdo con las inferencias normales de expectación, bien por el hablante o bien por el oyente, o incluso por otro participante de ese discurso. El acto locutivo ('primario', ya que no 'explícito', según la terminología de Austin) es propiamente una rectificación o una corrección, mientras que en (41 b) o (42 a) es una doble aseveración. No entraremos a analizar, sin embargo, cómo se articulan la promesa y la rectificación en oraciones como las de $(47 \mathrm{~b})$, porque nos interesa estudiar en particular los efectos sintácticos del peculiar semantismo de estas oraciones.

Las construcciones correctivas poseen unas características semánticas interesantes que tienen un claro reflejo sintáctico. Tales construcciones sólo resultan apropiadas si el sintagma sobre el que incide la negación en el segundo miembro de la coordinación o la yuxtaposición es el correspondiente al que constituye el foco en el primer miembro. Como es sabido, y simplificando, el foco de la oración es el constituyente que aporta la información nueva, por oposición al tema que especifica aquella entidad de la que predicaremos algo. En oraciones como (48)

(48) Juan confia en Luis.

existe una tendencia natural a interpretar Juan como tema y en Luis como foco (según algunos autores el rema sería confia en Luis, es decir, todo el predicado). Si queremos que Juan sea el foco de la oración, dicho sintagma habrá de recibir un acento intensivo. Nótese que dicho acento sobre Juan es insustituible en ( 49 a), pero no en (49 b)

(49) a. Juan confía en Luis, no Maria.

b. Juan confía en Luis, no en Maria.

Antes es para Bello una «conjunción correctiva" (\$1205) cuando equivale a antes bien, o a por el contrario, es decir, cuando urectifica una idea precedente». 
ya que en esta última oración el foco es en Luis y no Juan. Existe una tendencia general en español, señalada por varios autores, que nos lleva a situar el foco en posición final, o al menos postverbal. A la pregunta ¿Quién ha venido? no contestaremos probablemente Juan ha venido, sino $\mathrm{Ha}$ venido Juan, porque en la primera de estas dos oraciones, Juan se interpreta con mayor naturalidad como tema. A la pregunta ¿Qué ha ocurrido? podemos contestar indiferentemente Que Juan ha venido o Que ha venido Juan, porque la información nueva es ahora toda la oración, y no un único constituyente. Todos nuestros informantes coincidieron en que $(50 \mathrm{~b})$ es mucho menos natural que (50 a)

(50) a. Vino Juan, pero no Pedro.

b. ? Juan vino, pero no Pedro.

c. Juan vino, pero Pedro no.

Para que $(50$ b) fuera aceptable haría falta un marcadisimo acento intensivo sobre Juan, es decir, uno de los recursos (en este caso, fonético) que poseemos para identificar el foco con procedimientos formales.

Existen algunos recursos sintácticos para identificar el foco de la oración. Si la negación correctiva no incide sobre el sintagma paralelo al foco obtendremos en tales casos secuencias claramente agramaticales. Como el sujeto de una perífrasis de relativo identifica el foco, la oración (51 a) es correcta, pero (51 b) no lo es:

(51) a. Juan es el que confía en Luis, no María.

b. "Juan es el que confía en Luis, no en María.

Ciertas estructuras sintácticas, que algunos gramáticos llaman «dislocadas", identifican también el foco de la oración. Asi pues (52 a), es una oración correcta, pero (52 b) no lo es, como era de esperar, ya que el foco es vergüenza y no a ti.

a. Vergüenza debería darte, y no reparo.

b. *Vergüenza debería darte, y no a Juan.

Como hemos visto en (50 b) existe una cierta resistencia a interpretar, fuera de contexto, los sujetos preverbales como focos en español. Si estos sujetos están negados, aún es, si cabe, más difícil darles la interpretación citada. Necesitamos emplear el adverbio si para probar el contraste al que nos referimos: 
(53) a. Juan no vino, pero Pedro sí.

b. ?Juan no vino, pero sí Pedro.

c. No vino Juan, pero si Pedro.

Asi pues, (53 b) resulta forzada, porque Juan se interpreta como tema, y el constituyente ... si Pedro nos está exigiendo que lo interpretemos como foco. El problema desaparece en $(53 \mathrm{c})$ donde la posición de Juan nos permite interpretar dicho sintagma como foco con naturalidad.

Ya hemos comentado en el $\S 2$ que algunos verbos de afirmación permiten una ambigüedad interpretativa que rechazan otros. Veamos qué relación posee este fenómeno con el papel que el foco desempeña en la negación correctiva. Si comparamos las dos oraciones de (54):

(54) a. Juan dice que Pedro está enfermo, pero María no.

b. Juan dice que Pedro está enfermo, pero no María.

observaremos que es difícil interpretar el SN Juan en (54 b) como foco de dicha oración. Así pues, (54 b) no se interpretará en el sentido de "Maria no dice que Pedro está enfermo", salvo en un contexto en el que el SN Juan reciba un fuerte acento intensivo que le permitiera ser considerado foco. Si el sujeto no estuviera presente, por la consabida ausencia de los sujetos pronominales en español, seria imposible interpretarlo como foco. Así pues, la oración ( 55 a) no podría nunca ser interpretada, frente a (55 b), en el sentido de «Antonio no sintió que se marchara Pedro":

(55) a. Sentí que se marchara Pedro, no Antonio.

b. Senti que se marchara Pedro. Antonio no.

S. Kuno ha estudiado en varios trabajos (Kuno (1975), (1976a), (1976b), (1980) muchos de los efectos sintácticos que posee la oposición entre tema y foco, en particular en las estructuras elipticas. Los ejemplos siguientes, cuya versión inglesa pertenece a este autor, muestran claramente uno de estos efectos:

(56) a. ¿Vivias en un hotel en Paris?

b. ¿Compraste este cuadro en París?

Kuno observa correctamente (1980) (1976 b) que, si bien ambas oraciones pueden aceptar Si como única respuesta, es posible contestar Si, vivia en un hotel a la pregunta (56 a), pero no lo es responder Si, 
compré este cuadro a la pregunta (56 b). El sintagma en Paris (o su sustituto $a / / i$ ) se hace necesario en tal respuesta, pero no en la anterior (a menos, continúa Kuno, que se dé una interpretación, un tanto extraña, en la que comprar se use frente a robar, por ejemplo, de forma contrastiva). ¿Cuál es la causa de esta curiosa diferencia? La explicación la encontramos, siguiendo a Kuno, en el hecho de que en Paris es el foco en la oración (56 b) pero no lo es en (56 a). El determinante un de (56 a) frente a este de ( $56 \mathrm{~b}$ ) ayuda, evidentemente, a esta interpretación (pero no podemos detenernos a analizar este punto, que nos alejaria de nuestro objetivo). Si contestamos únicamente si a estas preguntas, este adverbio designa la información nueva, y no existe ningún problema. Ahora bien,si damos una contestación más larga, podemos suprimir en Paris en la respuesta a (56 a), porque dicho sintagma designa el 'tema' y, por tanto, ya ha sido introducido en el discurso, mientras que en (56 b) designa el 'foco', la información nueva, que no se puede suprimir dejando presente la conocida (= este cuadro). Por la misma razón, la respuesta Si, en Paris es apropiada como contestación a (56 b), y un tanto extraña como contestación a (56 a), ya que ello supondría que el interlocutor interpreta en Paris como foco en esa oración y no como tema, frente a lo que parece ser la interpretación habitual. Hemos visto que las negaciones correctivas únicamente inciden sobre el sintagma paralelo al que recibe la interpretación de 'foco'. Así pues, las diferencias que se observan en (57) y (58) son consecuencia lógica de este hecho. Señalamos en cursiva los sintagmas que se interpretan como focos:

(57) a. ¿Compraste este cuadro en Paris $\left\{\begin{array}{l}y \text { este otro no? } \\ \text { xy no este otro? }\end{array}\right\}$

b. ¿Vivias en un hotel en París $\left\{\begin{array}{l}\text { y no en casa de Pedro? } \\ \text { *y en casa de Pedro no? }\end{array}\right\}$

(58) a. ¿Compraste este cuadro en Paris $\left\{\begin{array}{l}y \text { en Barcelona no? } \\ \text { *y no en Barcelona? }\end{array}\right\}$

b. ¿Vivias en un hotel en Paris $\left\{\begin{array}{l}y \text { no en Barcelona? } \\ \text { *y en Barcelona no? }\end{array}\right\}$

Muy similar a la diferencia que existe entre las oraciones de (56) es la oposición entre las oraciones de (59), que pertenecen también a $\mathrm{S}$. Kuno:

(59) a. Juan nació en 1960.

b. Juan era todavía un bebé en 1960 . 
La oración (59 a) nos dice cuándo nació Juan (su foco es en 1960) mientras que (59 b) nos dice cómo era Juan en 1960 (la información nueva es era todavia un bebé). Kuno observa que si colocamos el SP en 1960 delante de las oraciones de (59), veremos que (59 a) cambia completamente de significado, ya que pasa a informarnos acerca de lo que ocurrió en 1960, en lugar de predicar algo de Juan. (59 b), en cambio, no altera su significado porque en 1960 constituye el tema (o al menos, parte de él) en dicha oración. El mismo autor compara los ejemplos de (60):

(60) a. ¿Nació Juan en 1960)

b. ¿Era Juan todavía un bebé en 1960 ?

y observa que respuestas como No, en 1961 son apropiadas para preguntas como (60 a), pero dificilmente lo serían para (60 b), ya que en 1960 se resiste a ser interpretado como foco en dicha oración. La posible respuesta $S i$, en 1960 muestra el mismo comportamiento que la anterior por idénticas razones. Nótese, además, que (60 b) podría contestarse con secuencias como Si exactamente eso, pero difícilmente con Si, exactamente entonces, a diferencia de (60 a).

Las oraciones en las que aparecen negaciones correctivas no son, como hemos visto, el resultado de procesos de elisión. Sin embargo, desde el punto de vista sintáctico poseen muchas propiedades formales en común con las elipsis parciales de SV en coordinadas ('gapping') a las que nos referiamos en los ejemplos de (20). De ello se debe deducir, en nuestra opinión, que la sintaxis de las negaciones correctivas está sujeta a principios formales de ámbito oracional que no poseen la relativa laxitud de los que regulan ciertas relaciones discursivas. Hankamer y Sag $(1976, \S 3.1)$ se refieren brevemente a estas estructuras $y$, aunque las consideran el resultado de un proceso de elipsis ('stripping'), frente a lo que nosotros proponemos para el español, admiten que tal regla «requires syntactic control». Veremos en los párrafos que siguen algunas de las relaciones formales que existen entre la negación correctiva y la elipsis parcial de SV en coordinadas ('gapping'):

a) Nótese, en primer lugar, que los constituyentes que permanecen en (20) tras las diversas elisiones ('remnants of gapping' en la terminologia generativista) son los mismos sobre los que incide la negación correctiva: 
(61) Juan piensa en irse de vacaciones a Italia este verano, y no

$$
\left\{\begin{array}{l}
\text { en irse de vacaciones a Francia este otoño. } \\
\text { a Francia este otoño. } \\
\text { este otoño. }
\end{array}\right\}
$$

Las variaciones que se observan en (61) son perfectamente compatibles con la posibilidad de identificar como foco un constituyente mayor o menor en el primer miembro de la coordinación.

b) Se ha observado ${ }^{9}$ que la elipsis parcial de SV en coordinadas sólo puede aplicarse a los constituyentes mayores, y no a los fragmentos de éstos, que también rechazan los procesos habituales de extracción. La misma conclusión puede aplicarse a las negaciones correctivas, como se observa en (62):

(62) a. Juan leyó el ejercicio de Pedro, y María $\varnothing$ el de Luis. $\lfloor\varnothing=$ leyó]

b. *Juan leyó el ejercicio de Pedro, y María, $\varnothing$ de Luis. $[\varnothing=$ leyó el ejercicio]

(63) Juan leyó el ejercicio de Pedro, pero no $\left\{\begin{array}{r}\text { el de } \\ \text { *de }\end{array}\right\}$ Luis.

La oración (62 b) es agramatical porque el ejercicio de Luis es un $\mathrm{SN}$ completo, y, como ya veíamos en (30), los procesos sintácticos afectan a la totalidad de estos constituyentes y no a alguna de sus partes. La simetria desaparece, sin embargo, en la yuxtaposición, donde es posible usar la negación correctiva en un sentido cercano al metalingüistico (Juan leyó el ejercicio de Pedro, no de Luis, con marcado acento intensivo sobre Pedro), pero aun en este caso sería de Pedro y no Pedro el constituyente que recibiría la negación correctiva. Nótese que $(64 \mathrm{c})$ es claramente agramatical en cualquier contexto:

(64) Se marcharon antes de las dos, y no $\left\{\begin{array}{ll}\text { a. } & \text { antes de las } \\ \text { b. } & \text { de las } \\ \text { c. } & \text { *las }\end{array}\right\}$ tres ya que es de las tres y no las tres el constituyente que desempeña una

9 Véase Hankamer (1973: 18) y Neijt (1979, \$§2.1.1, 2.2.1, 3.1.1, etc.). 
función dentro de una categoría mayor. Por idénticas razones nunca podrian ser sinónimas las dos oraciones de (49) ${ }^{\text {to }}$

c) También coincide la estructura sintáctica de las oraciones correctivas con la de las secuencias elípticas a las que nos referimos en la posibilidad de que sean varios los constituyentes que sigan a la conjunción, tanto si hay elipsis, como en (65a), como si no la hay, como en (65b):

(65) a. María invitó a Pedro a cenar, y Ana $\varnothing$ a Luis a comer.

b. María invitó a Pedro a cenar, y no a Ana a comer.

Existe una cierta polémica acerca de si el proceso de elipsis que reconocemos en (65a) es el mismo que el que corresponde a (62) 0 (20). Hankamer (1973) y A. Neijt (1979) piensan que sí, pero I. Sag (1977) piensa que no. En cualquier caso, oraciones como (65 b) coinciden con (46) y otras estructuras en mostrar que pueden ser varios los focos que se desean resaltar en el primer miembro de la coordinación. De hecho, el acento intensivo que identifica el foco puede recaer sobre varios constituyentes, como se observa en (66):

(66) María envió una carta a su jefe el martes, y no una postal a su novio el lunes.

d) La elipsis parcial de SV en coordinadas (gapping) afecta, como hemos visto en (62) y (20), a los constituyentes mayores, y no únicamente a sus complementos. Frente a la elipsis de SV que hemos estudiado en el $\S 2$, tales elisiones no pueden atravesar la barrera del subordinante, es decir, el nudo $\bar{\sigma}^{\prime 1}$ :

10 Para las construcciones como ¿Cuál $\varnothing$ es Juan? $\varnothing \varnothing=$ de ellos], que no analizaremos aqui, remitimos a Bosque (1983). Nótese que la elipsis de SP no es posible en el complemento de algunos adverbios interrogativos:

(i) a. Estaba satisfecho, pero no sé cómo de satisfecho.

b. "Estaba satisfecho pero no sé cómo $\varnothing \cdot[\varnothing=$ de satisfecho $]$.

"No podemos entrar a analizar con detalle las diferencias semánticas que existen entre las cláusulas correctivas introducias por y y las que introduce pero, porque ello nos adentraría en las complejisimas relaciones semánticas y pragmáticas de la coordinación adversativa. Sin embargo, debe tenerse en cuenta la sencilla regla introducida por Hurford (1974) para explicar oraciones como las de (i):

(i) a. Juan nació en Argentina, $\left\{\begin{array}{c}\text { pero } \\ * y\end{array}\right\}$ no en Buenos Aires.

b. Juan nació en Mendoza, $\left\{\begin{array}{c}\text { pero } \\ y\end{array}\right\}$ no en Buenos Aires. 
(67) a. Dijo que Luis quiere ir al monte y María $\varnothing$ a la playa.

b. *Dijo que Luis quiere ir al monte, y que María $\varnothing$ a la playa.

Las negaciones correctivas se comportan exactamente igual. La negación puede preceder a todo un constituyente oracional, como en (68 a), pero no puede incidir sobre uno de sus miembros si permanece el subordinante:

(68) a. Dijo que Luis queria ir al monte, y no que Maria quisiese ir a la playa.

b. Dijo que Luis quería ir al monte, y no (Maria) a la playa.

c. *Dijo que Luis quería ir al monte y no que Maria a la playa.

Existe una importante pregunta a la que todavia no hemos dado respuesta: Si las cláusulas que hemos llamado “correctivas" no son el resultado de un proceso de elisión, ¿cuál es entonces su estructura sintáctica? Creemos que la negación y el sintagma sobre el que incide constituyen una unidad sintagmática de clara función parentética. En nuestra opinión el adverbio modifica al sintagma al que precede y no a la conjunción en estructuras como las de (69)

(69) a. Veré a Juan, y no a Pedro.

b. Llegaron a las dos, y tal vez antes.

c. Conoció a Luis, y quizás a su mujer.

Fukuchi (1975) ha estudiado oraciones como Juan, y en consecuencia Pedro, ... y piensa que el SP o el adverbio modifica en ellas a la conjunción, y que entre ambos forman un "sintagma conjuntivo". Nos parece dificil adaptar esta idea a las oraciones de (69) porque en ellas puede desaparecer la conjunción sin alterar en absoluto la gramaticalidad. Al afirmar que cláusulas como y no a Pedro tienen en (69a) una función parentética, queremos indicar que su relación con la oración

c. Juan nació en Colombia, $\left\{\begin{array}{c}\text { "pero } \\ y\end{array}\right\}$ no en Buenos Aires.

La regla es la siguiente: Si reconstruimos la oración que obtendriamos en el segundo miembro de la coordinación aportando la información que falta, y obtenemos una oración que no implica la negación del primer miembro de la coordinación, la conjunción correcta es pero. En caso contrario será y. En efecto, Juan nació en Buenos Aires no implica la negación de Juan nació en Argentina y, por tanto, la conjunción pero es correcta en (i a), y la conjunción y es incorrecta en dicha oración. Sin embargo, Juan nació en Buenos Aires implica la negación de Juan nació en Mendoza, y también la de Juan nació en Colombia, luego la conjunción $y$ es la correcta, tanto en ( $i b$ ) como en (i c). 
que las precede es similar a la que existe entre creo yo y Juan lo sabe en Juan lo sabe, creo yo. Esta oración no es una simple transformación de Yo creo que Juan lo sabe, como muestran detenidamente Stillings (1975a) y Cornulier (1978), porque su estructura sintáctica responde a algunas características generales de las construcciones con incisos y no a la de una oración cuyo SV incorpore una subordinada sustantiva.

Desde el punto de vista sintáctico, las construcciones en las que el adverbio no precede a un sintagma no verbal pueden dividirse en dos grupos. En uno de ellos figuran aquellas que no necesitan de una interpretación semántica posterior que reconstruya ninguna información verbal, porque pueden ser modificadas libremente por el adverbio no: Vivia no lejos de aqui, Lo hizo no del todo mal. En el otro grupo figuran las negaciones correctivas, puesto que necesitan de dicho proceso de interpretación, cuya función es reconstruir, desde el punto de vista lógico, la información que se encuentra ausente en la estructura formal del enunciado. Dichas "reconstrucciones» o «interpretaciones semánticas" no se deben confundir con los procesos de elisión, que, por regla general, están sometidos a restricciones formales que no alcanzan a aquéllas. Desde el punto de vista estrictamente sintáctico, puede concluirse, por tanto, que no Juan es un constituyente en Vino Maria, no Juan, y que la información que se echa de menos entre el adverbio y el sustantivo no ha sido elidida por una regla sintáctica de supresión, sino que se reconstruye en un plano interpretativo claramente diferente del anterior.

Podemos confirmar que ( 41 a) no es un ejemplo de elipsis mientras que (41 b) si lo es. En esta última oración en Maria ocupa una posición temática idéntica a la que ocupa a Juan en $A$ Juan no lo vi. Esta oración, que algunos llaman «dislocada», como (52 a), no es el resultado de practicar alguna transformación en No vi a Juan, como muestra Rivero (1980). El SV elidido puede desempeñar una función similar a la del SV con el pronombre clítico: Vi a Pedro, pero a Juan no lo vi frente a $V i$ a Pedro, pero a Juan no $\varnothing$. Existe, por tanto, una elipsis de la forma confia tras el adverbio no en ( $41 \mathrm{~b}$ ), pero no en (41a).

4. Pueden señalarse al menos tres preguntas esenciales sobre la gramática de la elipsis que, sin ser las únicas posibles, deberian tener respuesta adecuada en los estudios, cada vez más necesarios, sobre esta categoria de la lengua. Su dificultad revela los enormes problemas que existen para abordar esa urgente gramática de las construcciones elípticas en español: 

A) ¿Qué fenómenos sintácticos son el resultado de los procesos formales de elisión y cuáles responden más bien a una interpre- tación o reconstrucción de la información ausente en un plano distinto del análisis?
B) ¿Cuáles son los contextos en los que las relaciones anafóricas se marcan por medio de pronombres y cuáles los que admiten la elipsis como exponente de tales relaciones?
C) ¿Qué procesos de elisión están controlados por factores sintác- ticos en el marco oracional y cuáles por factores pragmáticos o principios conversatorios?

Nos hemos referido a la pregunta $A$ en nuestro breve estudio de las negaciones correctivas en el epígrafe anterior. Algunos de los casos más claros, en apariencia, de elipsis controlada sintácticamente están siendo replanteados en la actualidad y tienden a verse más bien como el resultado de procesos interpretativos. Un ejemplo de ello lo tenemos en la elipsis que se muestra en (70), estudiada detenidamente por Ross (1969)

(70) Juan habló con alguien, pero no sé con quién $\varnothing$. $\varnothing \varnothing=$ habló Juan]

L. Levin (1982) observa que si entendiéramos estas estructuras como el resultado de un proceso sintáctico de elisión, habriamos de partir de secuencias claramente agramaticales porque violan principios básicos de la sintaxis. Fijémonos en las oraciones de (71):

(71) a. Me hablaron de la posibilidad de entrevistarme con alguien, pero no recuerdo con quién $\varnothing$.

b. Pedro me preguntó dónde compró Luis uno de sus cuadros, pero no recuerdo cuál $\varnothing$.

Si interpretáramos estas oraciones como productos de un proceso formal de elipsis, deberiamos suponer que con quién $\varnothing$ en (71 a) deriva de *con quién me hablaron de la posibilidad de entrevistarme, y que cuál $\varnothing$ en $(71 \mathrm{~b})$ deriva de "cuál cuadro me preguntó Pedro dónde compró Luis. Es muy improbable, argumenta Levin, que éstos sean procesos sintácticos de elisión. La información que aportan puede obtenerse, en cambio, mediante interpretaciones semánticas que no obedezcan las rigurosas constricciones del plano sintáctico. Algunos autores proponen que tales interpretaciones se realicen en el plano del análisis que se suele llamar "forma lógica», pero, en cualquier caso (y 
aunque ello sirva de escaso consuelo), la pregunta $A$ tampoco ha recibido una única respuesta definitiva en las gramáticas de otras lenguas.

La pregunta $B$ es, probablemente, la que está sujeta a mayor variación comparando los sistemas gramaticales de los diferentes idiomas. Así, por ejemplo, el vasco y casi todas las lenguas germánicas (entre otras muchas) figuran entre los idiomas que eliden ciertos constituyentes que se pronominalizan en las lenguas romances. (No existe forma alguna que se corresponda con el pronombre to en las oraciones equivalentes a Juan no lo es en dichas lenguas.) En español existe una compleja combinación de factores que regulan la alternancia 'elipsis/ pronombre'. Aunque pensamos dedicar un próximo trabajo únicamente a la pregunta $B$, señalaremos aquí que entre esos factores figura el significado 'específico' o 'no específico' del SN que va a ser objeto de referencia anafórica, como se observa en (72) y (73):

(72) A ¿Tienes un bolígrafo?

$B_{1}$ No, no tengo $\varnothing$.

$B_{2}$ "No, no lo tengo.

(73) A ¿Tienes un boligrafo mío?

$B_{1}$ "No, no tengo $\varnothing$.

$B_{2}$ No, no lo tengo.

así como el carácter 'contable' o 'no contable' del sustantivo, como se observa en (74)
a. Luis tenía dinero, pero Antonio no
$\left\{\begin{array}{l}\text { tenía } \varnothing \\ \text { ?lo tenía }\end{array}\right\}$
b. A María le dio pena, pero a Juan no
$\left\{\begin{array}{l}\text { le dio } \varnothing \\ \text { "se la dio }\end{array}\right\}$

y la naturaleza léxica del verbo cuyo complemento va a ser objeto de referencia anafórica, como se observa en (75)

(75) A ¿Quieres un güisqui?

$B_{1}$ No, no quiero $\varnothing \cdot[\varnothing=$ un güisqui $]$

$\mathrm{B}_{2}$ No, no bebo.

$\mathrm{B}_{3}{ }^{*}$ No, no bebo $\varnothing \cdot[\varnothing=$ güisqui $]$

En cuanto a la pregunta $C$, nos hemos referido brevemente a ella en el párrafo que sigue al ejemplo (17). La respuesta es esencial para 
contribuir de forma significativa a establecer la línea de separación entre la gramática de la oración y la del discurso. El artículo citado de Hankamer y Sag constituye un buen punto de partida en esa direción por lo que respecta al estudio de la elipsis. Las elisiones son, según se suele afirmar, procesos recuperables, porque la información que se elide se debe reconocer en la que permanece. Sin embargo, las elisiones controladas pragmáticamente muestran que no sólo se elide lo que se repite verbalmente. La 'recuperación' (por usar el término técnico habitual) de la información ausente se realiza, con frecuencia, atendiendo a un cierto conocimiento extralingüístico que comparten el hablante y el oyente.

Existen muchas clases de elipsis o, dicho de otra forma, el término 'elipsis' designa fenómenos muy diferentes. Ninguna de las tres preguntas que aquí se recogen tiene una respuesta sencilla, pero ninguna de ellas debería quedar tampoco, en nuestra opinión, fuera de los intereses del gramático.

\section{Referencias bibliográficas}

Bello, Andrés (1847), Gramática de la lengua castellana, citamos por la edición de R. Trujillo, Tenerife, 1981.

Bosque, Ignacio (1980), Sobre la negación, Madrid, Cátedra.

- (1983), «La selección de las palabras interrogativas", en prensa en Verba (Santiago de Compostela).

Carbonero, Pedro (1980), «Afirmación, negación, duda», en Revista Española de Lingüistica, 10, 2, pp. 161-175.

Cornulier, B. de (1978), "L'incise, la classe des verbes parenthétiques et le signe mimique", en Cahiers de Linguistique, Quebec, 8, pp. 53-97.

Dumitrescu, Domnita (1973), "Apuntes sobre el uso enfático de si (adv.) en el español contemporáneo", en Revue Roumaine de Linguistique, 18, 5, pp. 407-413.

- (1979), "El sistema de las respuestas minimales en castellano", en Revue Roumaine de Linguistique, 24, 1, pp. 45-54.

Fukuchi, Hajame (1975), "Remarks on the Adverbials in Coordinate Structure», en Papers in Linguistics, 8, 1-2, pp. 177-197.

Hankamer, Jorge (1971), Constraints on Delation in Syntax, tesis de la Universidad de Yale publicada en 1979 por Garland, Nueva York. 
- (1973), «Unacceptable Ambiguity», en Linguistic Inquiry, 4, pp. 17-68.

- y Sag, Ivan (1976), «Deep and Surface Anaphora», en Linguistic Inquiry, 7, 3, 1976, 391-426.

Hooper, Joan (1974), "On Assertive Predicates", Indiana University Linguistics Club.

Hurford, J. R. (1974), "Exclusive or Inclusive Disjunction», en Foundations of Language, 11, págs. 409-411.

Jackendoff, Ray (1971), "Gapping and Related Rules», en Linguistic Inquiry, 2, pp. 21-35.

Kato, Mary (1981), «Deletion in Coordinate Structures in Portuguese", en W. C. Cressey y D. J. Napoli (eds.), Linguistic Symposium on Romance Languages: 9, Washington, Georgetown University Press, pp. 308-330.

Kuno, Susumo (1975), “Conditions for Verb Phrase Deletion», en Foundations of Language, 13, pp. 161-175.

- (1976a), "Gapping: A Functional Analysis", en Linguistic Inquiry, 7, 2, pp. 300-318.

- (1976b), «Three Perspectives in the Functional Study of Syntax», en L. Matejka (ed.), Sound, Sign and Meaning. Quinquagenary of the Prague Linguistic Circle, Ann Arbor (Michigan), pp. 119-190.

- (1980), «Functional Syntax», en E. A. Moravsick y J. R. Wirth (eds.), Current Approaches to Syntax, N. York, Academic Press, pp. 117-135.

- (1982), "The Focus of the Question and the Focus of the Answer», en Papers from the Parasession on Nondeclaratives, Chicago Linguistic Society, págs. 134-157.

Lázaro Mora, Fernando (1982), "Sobre aunque adversativo", en Lingüistica Española actual, 4.1, pp. 123-130.

Levin, Lori (1982), “Sluicing: A Lexical Interpretation Procedure», en J.

Bresnan (ed.), The Mental Representation of Grammatical Functions, Cambridge, MIT, pp. 590-654.

Lysvag, Per (1975), "Verbs of Hedging», en J. Kimball (ed.), Syntax and Semantics -4, N. York, Academic Press, pp. 125-154.

Martínez Alvarez, Josefina (1971), «Poder + infinitvo», en Archivum, 27-28, pp. 397-414.

Narbona, Antonio (1981), “¿Verbos modales en español?», en Verba, 8, pp. 171-186.

Neijt, Anneke (1979), Gapping. A Contribution to Sentence Grammar, Dordrech, Foris Publications.

Plantin, Christian (1982), "Oui et non sont-ils des 'pro-phrases'?", en Le français moderne, 50,3 , pp. 252-265. 
Rivero, M. ${ }^{a}$ Luisa (1980), "On Left Dislocation and Topicalization in Spanish», en Linguistic Inquiry, 11, 2, pp. 363-393.

Ross, John (1967), "Gapping and the Order of Constituents", en M. Bierwisch y K. Heidolph (eds.), Progress in Linguistics, La Haya, Mouton, 1970, pp. 250-259.

- (1969), "Guess Who», en R. Binnick y otros (eds.), Papers from the Fifth Regional Meeting of the Chicago Linguistic Society, pp. 252-286.

Sag, Ivan (1977), Deletion and Logical Form, Indiana University Linguistics Club.

Stillings, Justine (1975), "The Formulation of Gapping as Evidence for Variable Types in Syntactic Transformations», en Linguistic Analysis, 1, pp. 247-273.

- (1975a), "Sentence Raising», Indiana University Linguistics Club.

Urmson, J. (1952), “Parenthetical Verbs», en Mind, 61, pp. 48-496. Citamos por la reproducción que aparece en C. E. Caton (ed.), Philosophy and Ordinary Language, Urbana, University of Illinois Press, 1964, pp. 220-240.

Williams, Edwin (1977), "Discourse and Logical Form», en Linguistic Inquiry, 8, 1, pp. 101-139. 